\title{
DETECCIÓN DE HONGOS TOXIGÉNICOS EN LA CADENA PRODUCTIVA DE LA MACA (Lepidium meyenii Walp)
}

\section{DETECTION OF TOXIGENIC FUNGI IN THE PRODUCTIVE CHAIN OF THE MACA}

\section{(Lepidium meyenii Walp )}

Emilio Fredy Yábar Villanueva', Vilma Julia Reyes De la Cruz'

\section{RESUMEN}

Los principales géneros de hongos potencialmente toxigénicos en la cadena productiva de la maca son el Fusarium, Penicillium, Aspergillus y Rhizopus, el primero procedente del campo y los restantes durante su manipulación poscosecha. La humedad de la maca fresca, es precursor de su presencia en la cadena productiva. La humedad en las harinas de maca cruda, gelatinizada y precocida inhiben su crecimiento sin embargo los tratamientos tecnológicos no garantizan su ausencia en el producto. Los azúcares reductores en la maca y derivados son el principal sustrato y el $\mathrm{pH}$ en ellos es óptimo para el crecimiento de los hongos.

Palabras Clave: Hongos toxigénicos, Fusarium, Penicillium, Aspergillus

\begin{abstract}
The main toxigenic genera of fungi in the productive chain of maca are Fusarium, Penicillium, Aspergillus and Rhizopus. The first one was found pre harvest and the rest during post harvest manipulation. The moisture of fresh maca is the precursor of this fungi presence in productive chain of maca. The moisture of the maca powder, gelatinized maca flour, and precooked maca flour inhibited their growth however the technological treatment doesn't guarantee the absence of fungi in the product. The reducing sugars in the products made of maca are the main substrates and the $\mathrm{pH}$ in them is optimum for the fungi growth.
\end{abstract}

Key words: Toxigenic fungi, Fusarium, Penicillium, Aspergillus 


\section{INTRODUCCIÓN}

El trabajo de investigación, tuvo como objetivos, identificar los hongos toxigénicos presentes en la cadena productiva de la maca. En la cadena agroalimentaria es importante estudiar su calidad desde un punto de vista multidisciplinario e integral, debido a que permite resolver los problemas en forma consistente.

La presencia de hongos toxigénicos en cualquier producto alimenticio siempre han constituido un peligro y son de mucho riesgo para la salud, debido sobre todo a que dichos microorganismos producen metabolitos secundarios denominados micotoxinas, los cuales, por estudios recientes, están demostrando que tienen efecto mutágeno, teratógeno, cancerígeno, hepatotóxico, nefrotóxico, neurotóxico, citotóxico y endocrino, que no tienen un efecto inmediato sino que se presentan después de un cierto período de tiempo, (1).

\section{MATERIAL Y MÉTODOS}

La investigación se realizó en el Laboratorio de Microbiología de Alimentos de la Universidad Nacional del Centro del Perú. Se trabajó con maca del ecotipo blanca procedente de la provincia de Junín y de los mercados de abasto de la ciudad de Huancayo y Chupaca en sus diferentes formas de presentación como: maca fresca y seca, harinas de maca cruda, gelatinizada y precocida a los cuales se realizaron controles fisicoquímicos (2) y microbiológicos (3) para identificar factores de crecimiento, cuantificación de hongos filamentosos y su respectiva identificación.

\section{RESULTADOS}

\section{Controles Fisicoquímicos}

\section{Humedad}

La tabla 1, muestra la determinación de la humedad en la cadena productiva de maca. La humedad es una variable preponderante para el desarrollo de los hongos de campo y de almacenamiento.

Tabla 1. Contenido de humedad en la caden a uroductiva de la larina

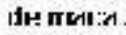

\begin{tabular}{|c|c|}
\hline Proceso de elaboración de harina de maca & llumedad $(\mathrm{X})$ \\
\hline $\begin{array}{l}\text { Mele its;A } \\
\text { Mac:a iค:a }\end{array}$ & $\begin{array}{l}68,37 \\
15.12\end{array}$ \\
\hline Harir $=$ da ma: acr & y $y_{1}$ \\
\hline Harir $\mathrm{d}$ d maz a gelxin בada & $6>0$ \\
\hline I larir $\varepsilon$ de mas a precoc ca & 537 \\
\hline
\end{tabular}

\section{Azúcares Reductores}

La tabla 2, muestra la determinación de la humedad en la cadena productiva de maca. Los azúcares reductores son los principales sustratos y necesarios para el desarrollo de los hongos de campo y de almacenamiento.

\section{pH}

abla 2 Contenido de azúcares reductores en la cadena productiva de la harina de maca

Proceso de elaboración de harina de maca

Azúcares reductores (16)

Mar: 7 rescis

$1,2 \mathrm{hh}$

12,613

14,164

17,275

18,105
La tabla 3, muestra la determinación del pH en la cadena productiva de maca. El pH es un factor físico incondicional para el desarrollo de los hongos de campo y de almacenamiento.

I abla 3. pH en I cadenaproducina de Ia harına de maca

\begin{tabular}{ll} 
Proceso de elaboración de harina de maca & $\boldsymbol{\mu H}$ \\
\hline Vac a tresca & 5,65 \\
Mac a seca & 5,07 \\
Jarira de maca cruya & $5,6 \mathrm{C}$ \\
Jarlra de meca gelatnlzaja & $5,6 \mathrm{C}$ \\
larira de meca precocica & $5,1 \mathrm{C}$
\end{tabular}

\section{Controles Microbiológicos}

Recuento de Hongos en la cadena Productiva de Maca

La tabla 4, muestra los recuentos de hongos en la cadena productiva de harina de maca cruda. 
Tabla 1. Recuento de hongos en la cadena productiva de harina de maca.

\begin{tabular}{|c|c|c|c|c|c|}
\hline ETAPA & $\begin{array}{l}\text { No Total dc } \\
\text { co onlas de } \\
\text { hongos } \\
\text { ufici. }\end{array}$ & $\begin{array}{l}\text { Fusorum } \\
\text { Ursig }\end{array}$ & $\begin{array}{l}\text { Ponicilium } \\
\text { urcig }\end{array}$ & $\begin{array}{l}\text { Asperginus } \\
\text { utcrg }\end{array}$ & $\begin{array}{l}\text { Rhizo, } \\
\text { urcig }\end{array}$ \\
\hline N aca 1res:a & $14 \times 10^{2}$ & $2 \times 10^{2}$ & - & $8 x^{\prime} 0^{2}$ & $6 \times 10$ \\
\hline N aca seca & $30 \times 10$ & $1 \times 10$ & $2 \times 10$ & $1 \times 10$ & 'Ex1L \\
\hline $\begin{array}{l}\text {-a'lna ce } \\
\text { maca cruda }\end{array}$ & $35 \times 10$ & - & $2 \times 10$ & $12 \times 13$ & $9 \times 0$ \\
\hline $\begin{array}{l}\text {-a'ina ce } \\
\text { maca } \\
\text { gelatinizad }\end{array}$ & $3 x \cdot 0$ & - & $1 \mathrm{C}$ & $2 \times 10$ & - \\
\hline $\begin{array}{l}\text {-aina ce } \\
\text { maca } \\
\text { prec ocica }\end{array}$ & $12 \times 10$ & $2 \times 10$ & $4 \times 10$ & $6 \times 10$ & $8 \times 1 \mathrm{~J}$ \\
\hline
\end{tabular}

\section{DISCUSIÓN}

Los hongos requieren de ciertos factores para la regulación de su crecimiento y desarrollo, uno de los

\section{Aislamiento e Identificación de Hongos Presentes en la Maca}

factores extrínsecos importantes es la humedad del medio en general mayores a $17 \%$ y una Aw superior a 0,85 (4). La Humedad en la maca fresca es en promedio $68,37 \%$ y seca $15,12 \%$, condición favorable para los hongos, son precursores de la presencia de hongos potencialmente toxigénicos en la cadena productiva de maca. Los contenidos de humedad en la harina de maca cruda de 9,87\%, harina de maca gelatinizada de $6,40 \%$ y harina de maca precocida de $5,37 \%$ inhiben el crecimiento de los hongos y los tratamientos tecnológicos a los que son sometidos no garantizan su ausencia en el producto $(2,4)$. El pH aunque variado en muchas especies, por lo general debe mantenerse entre los 4 a 6 y la maca tiene un $\mathrm{pH}$ de 5,18 a 5,67 en toda su cadena productiva. Los azúcares reductores presentes en la maca fresca de $1,256 \%$, maca seca $12,618 \%$, harina de maca cruda 14,164\%, harina de maca gelatinizada $17,276 \%$ y harina de maca precocida $18,105 \%$, son los principales sustratos alimenticios para los hongos toxigénicos.

Aspsmans

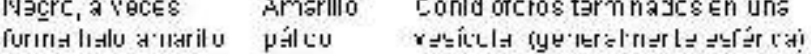

vede inor, con surenlic $\theta$. Esporas ar cadjra desje el

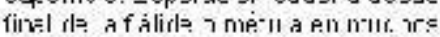
colores Jcpencisnds ds a copccis.
Espวrar gic ( $5 a_{c o}$ ) ccmen ende esporas
Hizhopls

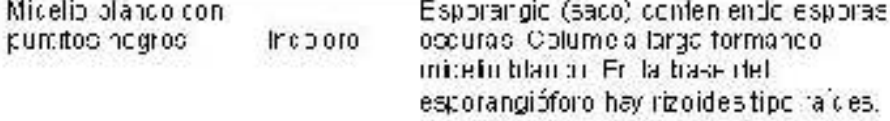

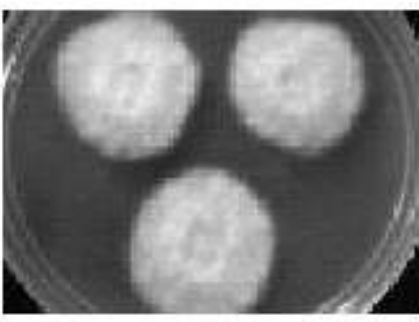

Futografia 1. Colonias de Fusarium en DGA. $12^{\circ} \mathrm{C}$ facilitando el crecimiento de Fusarium y Penicillium y en los mercados un poco más elevada hasta $20^{\circ} \mathrm{C}$ facilitando el crecimiento y desarrollo de Aspergillus.

Se han identificado los siguientes hongos toxigénicos según las guías de identificación de hongos (6) Fusarium, Penicilllium y Aspergillius. 
Las siguientes fotografías corresponden a los principales géneros de hongos potencialmente toxigénicos presentes en la cadena productiva de maca.

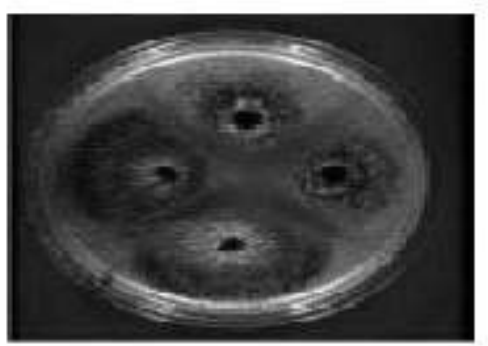

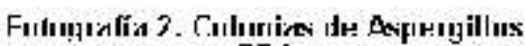
en РПА.

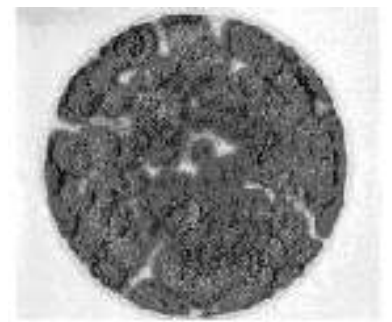

Fotagrafía O. Calonias tle Penicillium en OGA.

\section{REFERENCIAS BIBLIOGRÁFICAS}

1. Derache, R.. Toxicología y Seguridad de los Alimentos. Edit. OMEGA, S.A. Barcelona; 1990.

2. Ponce, C.. Elaboración de una Conserva a Base de Maca (Lepidium meyenii Walp) Utilizando Envases de Vidrio. Tesis para Optar el Título Profesional de Ingeniero en Industrias Alimentarias. UNCP-Huancayo-Perú; 1999

3. ICMSF Microorganismos de los Alimentos Técnicas de análisis microbiológico Vol. I 2da Ed. Editorial ACRIBIA Zaragoza España; 1983.

4. ICMSF Microorganismos de los AlimentosCaracterísticas de los patógenos microbianos. Editorial ACRIBIA S.A. Zaragoza- España; 1996

5. Aliaga Cárdenas, R. Guía para el cultivo, aprovechamiento y conservación de la maca, Lepidium meyenii walpers- Convenio Andrés Bello, Serie Ciencia y Tecnología No 82 SECAB Lima; 1999.

6. Pitt, J.I. and Hocking A.d.. fungi and food spoilage. Sydney: Academic Press EEUU; 1985

E-mail:vilmareyes23@latinmail.com 